Supporting Information:

\title{
A First-Principles Study of Impurity-Enhanced Adhesion and Lubricity of Graphene on Iron Oxide Surface
}

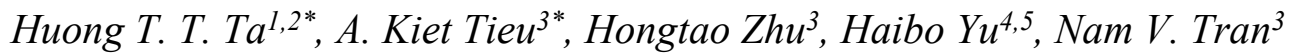 \\ ${ }^{1}$ Phenikaa Institute for Advanced Study (PIAS), Phenikaa University, Yen Nghia, Ha Dong, \\ Hanoi 12116, Vietnam. \\ ${ }^{2}$ Faculty of Materials Science and Engineering, Phenikaa University, Yen Nghia, Ha Dong, \\ Hanoi 12116, Vietnam. \\ ${ }^{3}$ School of Mechanical, Materials, Mechatronic and Biomedical Engineering, University of \\ Wollongong, Northfield Avenue, Wollongong, NSW 2522, Australia. \\ ${ }^{4}$ School of Chemistry and Molecular Bioscience, University of Wollongong, Northfield \\ Avenue, Wollongong, NSW 2522, Australia. \\ ${ }^{5}$ Molecular Horizons, University of Wollongong, Northfields Avenue, Wollongong, NSW \\ 2522, Australia.
}

*Corresponding author:huong.tathithuy@phenikaa-uni.edu.vn (Huong T. T. Ta)

ktieu@uow.edu.au (A. Kiet Tieu) 


\section{Optimization of the lattice constants and structures for doped-graphene}
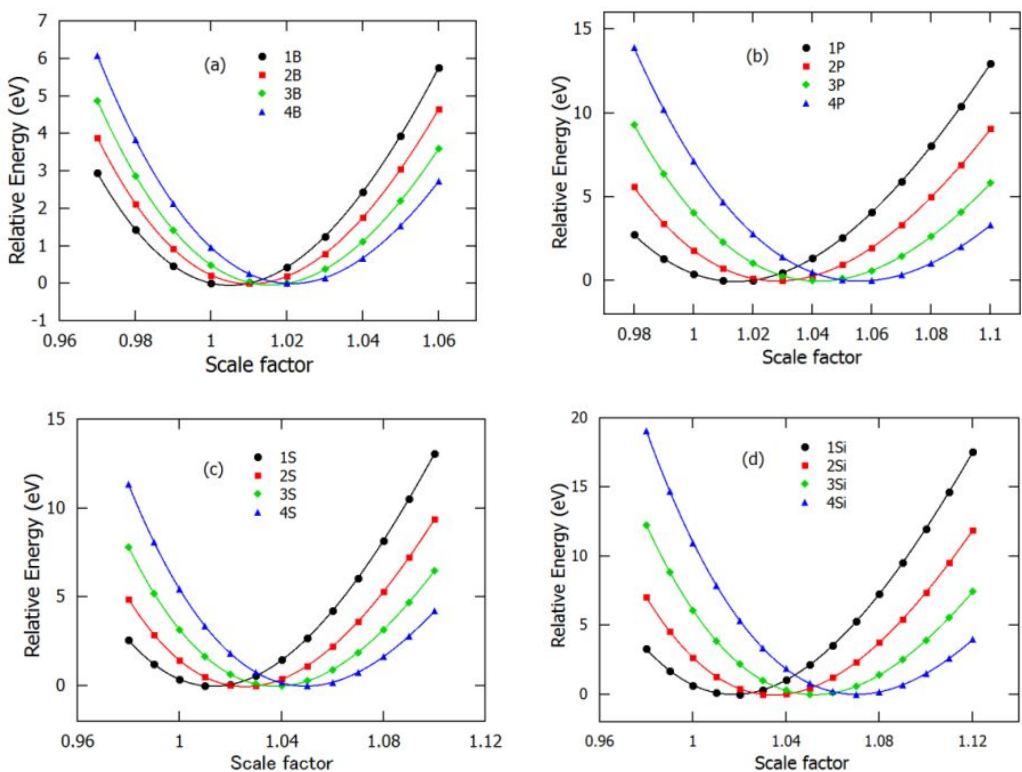

Figure S1. Optimization of the lattice constants for DG structures with the number of doping atoms: (a) BG, (b) PG, (c) SG, and (d) SiG. The original graphene lattice is given the scale factor 1.0 as a reference.

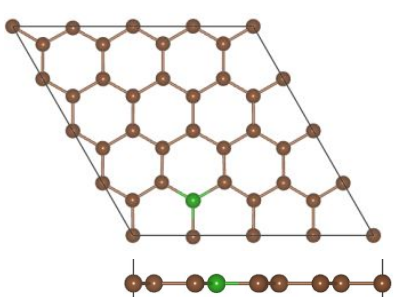

(a) $1 \mathrm{BG}$

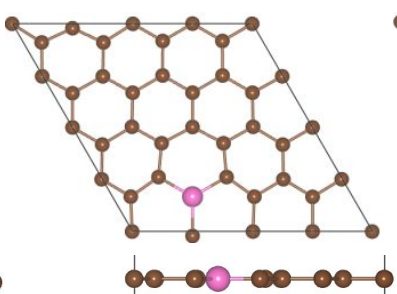

(b) $1 \mathrm{PG}$

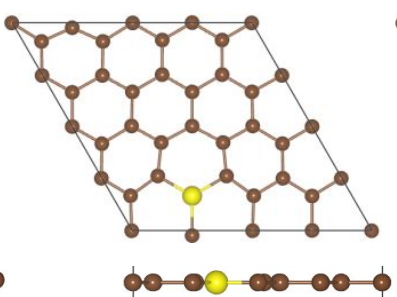

(c) $1 \mathrm{SG}$

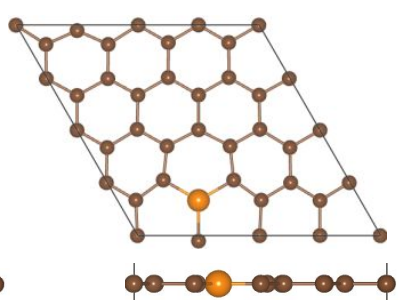

(d) $1 \mathrm{SiG}$

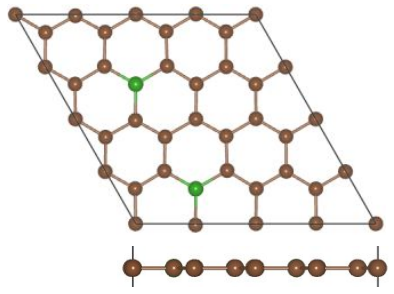

(e) 2BG

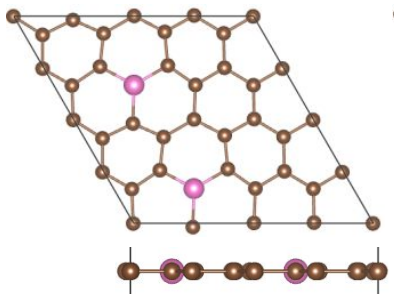

(f) $2 P G$

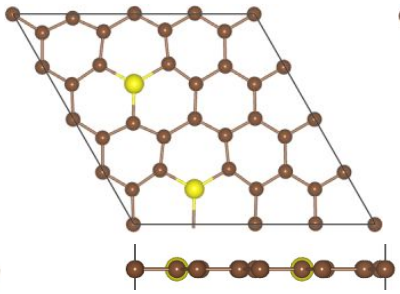

(a) 2 SG

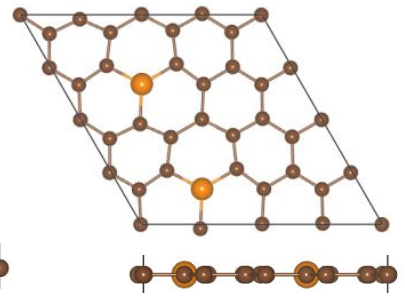

(h) $2 \mathrm{SiG}$

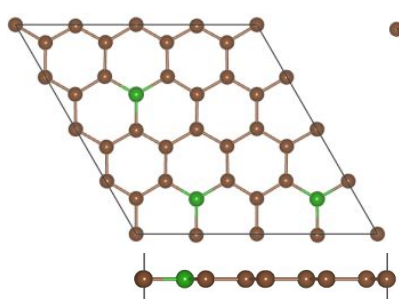

(i) 3BG

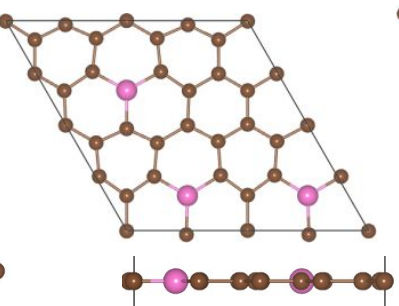

(i) 3PG

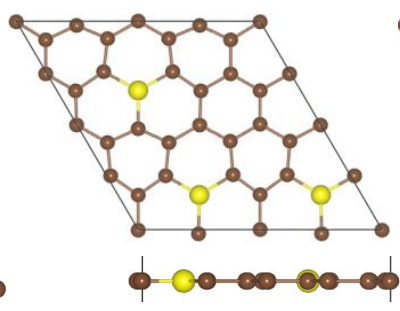

(k) 3 SG

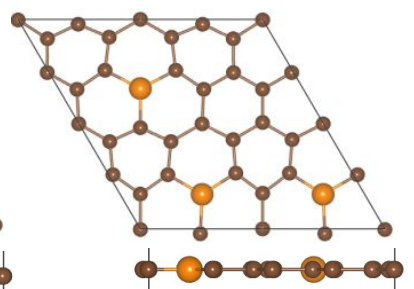

(I) $3 \mathrm{SiG}$

Figure S2. Top view and side view of the optimized configurations of 1DG, 2DG, and 3DG. 


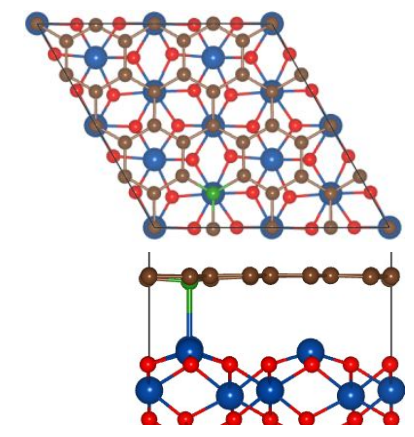

(a) Fe1BG

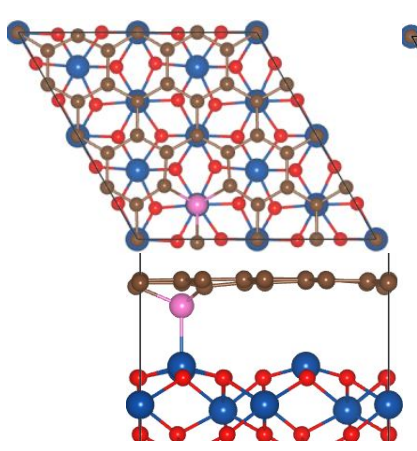

(b) Fe1PG

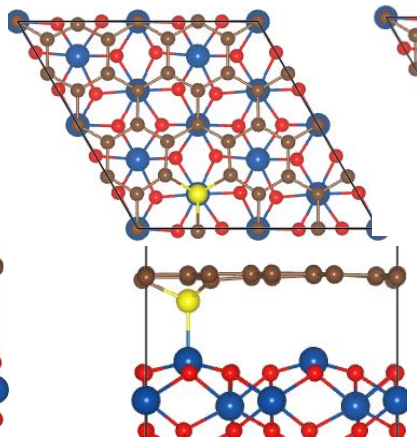

(c) Fe1SG

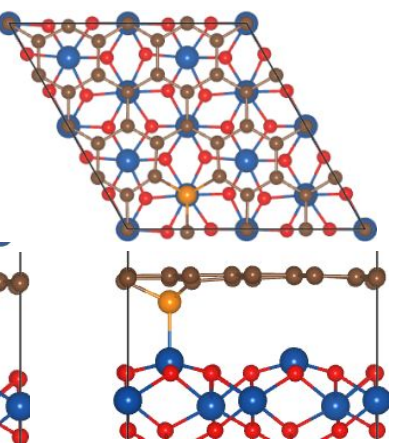

(d) $\mathrm{Fe} 1 \mathrm{SiG}$

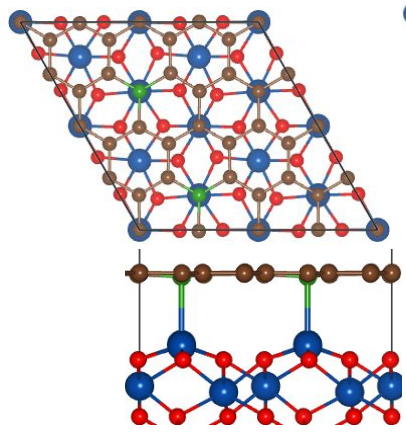

(e) Fe2BG

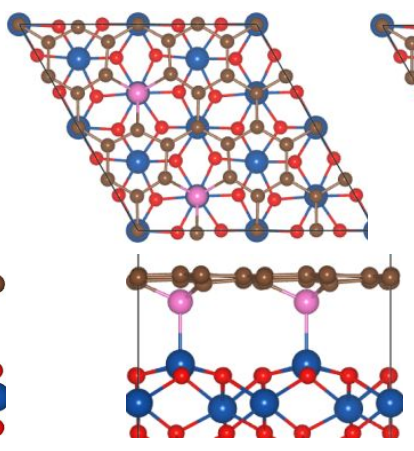

(f) Fe2PG

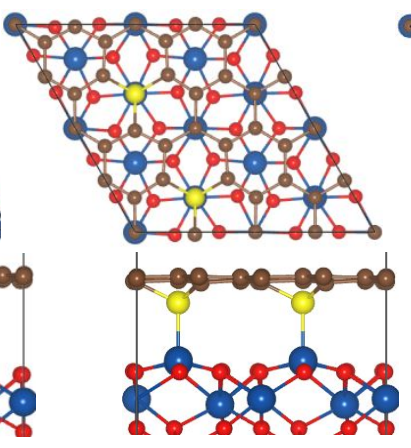

(g) Fe2SG

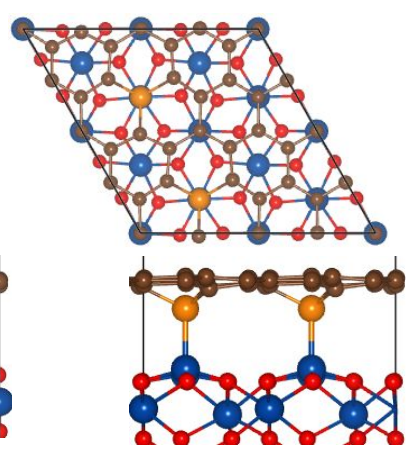

(h) Fe2SiG

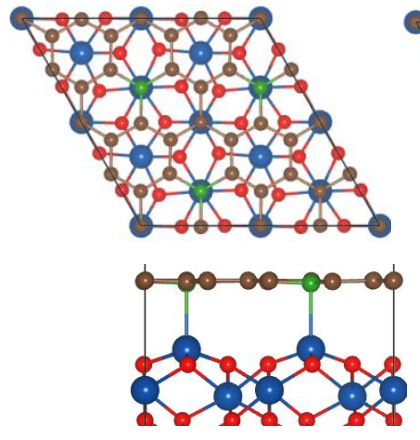

(i) Fe3BG

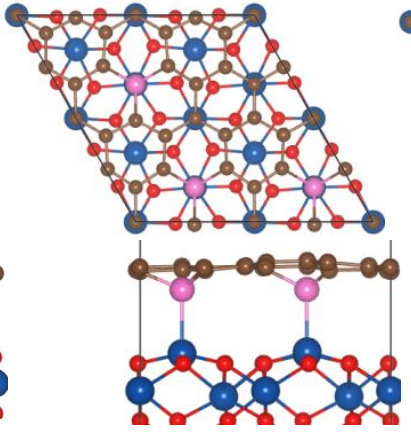

(i) $\mathrm{Fe} 3 \mathrm{PG}$

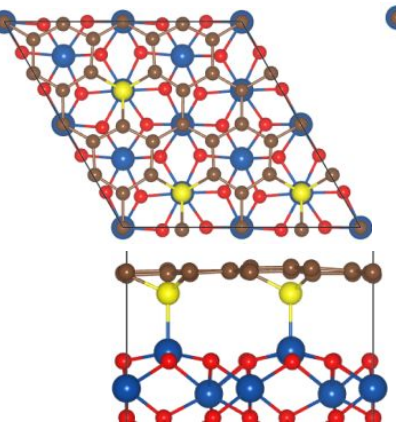

(k) Fe3SG

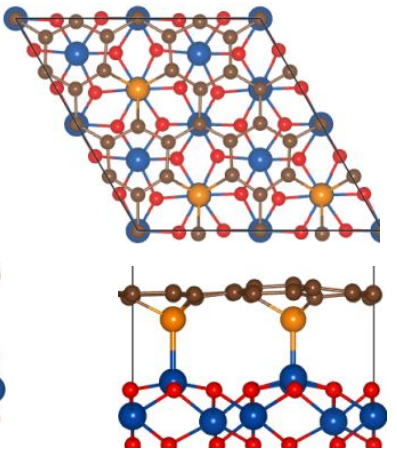

(I) Fe3SiG

Figure S3. Top view and side view of the optimized configurations of Fe1DG, Fe2DG, and Fe3DG.

\section{PES}

The binding energy of two slabs was calculated by $E_{b}=\left(2 E_{\text {slab }}-E_{\text {tot }}\right) / N$, where $E_{\text {slab }}$ is the total energy of one separate slab, $E_{\text {tot }}$ is the total energy of the whole system, and $\mathrm{N}$ is the total number of atoms in the system. 
Table S1. Binding energy of the maximun $\left[\mathrm{E}_{\mathrm{b}}(\max )\right]$ of minimum $\left[\mathrm{E}_{\mathrm{b}}(\min )\right]$ configurations, barier energy $\left[\Delta \mathrm{E}=\mathrm{E}_{\mathrm{b}}(\max )-\mathrm{E}_{\mathrm{b}}(\min )\right]$, and equilibrium distance between two substrates of the maximun $\mathrm{D}_{\mathrm{eq}}(\max )$ and minumum $\left(\mathrm{D}_{\mathrm{eq}}(\min )\right.$ configurations.

\begin{tabular}{lccccc}
\hline \hline System & $\begin{array}{c}\mathrm{E}_{\mathrm{b}}(\min ) \\
(\mathrm{meV} / \text { atom })\end{array}$ & $\begin{array}{c}\mathrm{E}_{\mathrm{b}}(\max ) \\
(\mathrm{meV} / \text { atom })\end{array}$ & $\begin{array}{c}\Delta \mathrm{E} \\
(\mathrm{meV} / \text { atom })\end{array}$ & $\begin{array}{c}\mathrm{D}_{\text {eq }}(\min ) \\
(\AA)\end{array}$ & $\begin{array}{c}\mathrm{D}_{\text {eq }}(\max ) \\
(\AA)\end{array}$ \\
\hline $\mathrm{Fe}_{2} \mathrm{O}_{3}$ & 173.10 & 113.84 & 59.27 & 1.39 & 2.17 \\
$\mathrm{FeG}$ & 125.56 & 122.82 & 2.74 & 3.53 & 3.66 \\
\hline $\mathrm{Fe} 1 \mathrm{BG}$ & 128.41 & 125.38 & 3.03 & 3.36 & 3.47 \\
$\mathrm{Fe} 2 \mathrm{BG}$ & 125.27 & 122.55 & 2.72 & 3.36 & 3.47 \\
$\mathrm{Fe} 3 \mathrm{BG}$ & 122.30 & 120.00 & 2.30 & 3.33 & 3.38 \\
$\mathrm{Fe} 4 \mathrm{BG}$ & 120.15 & 118.07 & 2.06 & 3.31 & 3.37 \\
\hline $\mathrm{Fe} 1 \mathrm{PG}$ & 121.92 & 118.87 & 3.05 & 3.37 & 3.45 \\
$\mathrm{Fe} 2 \mathrm{PG}$ & 119.58 & 116.72 & 2.86 & 3.21 & 3.42 \\
$\mathrm{Fe} 3 \mathrm{PG}$ & 115.04 & 113.00 & 2.02 & 3.15 & 3.23 \\
$\mathrm{Fe} 4 \mathrm{PG}$ & 113.47 & 112.00 & 1.46 & 3.36 & 3.49 \\
\hline $\mathrm{Fe} 1 \mathrm{SG}$ & 125.14 & 121.88 & 3.27 & 3.37 & 3.46 \\
$\mathrm{Fe} 2 \mathrm{SG}$ & 123.92 & 120.24 & 3.68 & 3.24 & 3.37 \\
$\mathrm{Fe} 3 \mathrm{SG}$ & 122.83 & 119.27 & 3.56 & 3.23 & 3.44 \\
$\mathrm{Fe} 4 \mathrm{SG}$ & 108.16 & 102.77 & 5.39 & 3.29 & 3.45 \\
\hline $\mathrm{Fe} 1 \mathrm{SiG}$ & 119.01 & 116.16 & 2.85 & 3.28 & 3.40 \\
$\mathrm{Fe} 2 \mathrm{SiG}$ & 103.46 & 100.26 & 3.20 & 3.05 & 3.26 \\
$\mathrm{Fe} 3 \mathrm{SiG}$ & 101.56 & 96.91 & 4.65 & 2.87 & 3.00 \\
$\mathrm{Fe} 4 \mathrm{SiG}$ & 100.22 & 94.94 & 5.27 & 3.10 & 3.17 \\
\hline \hline
\end{tabular}

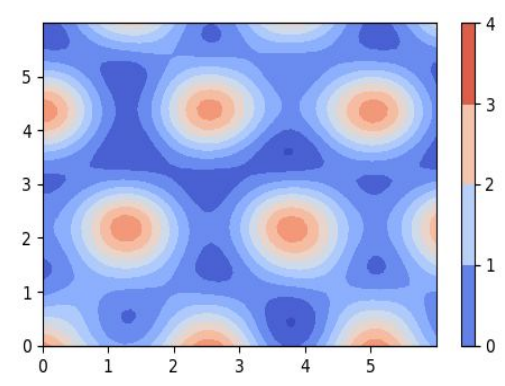

(a) Fe1PG

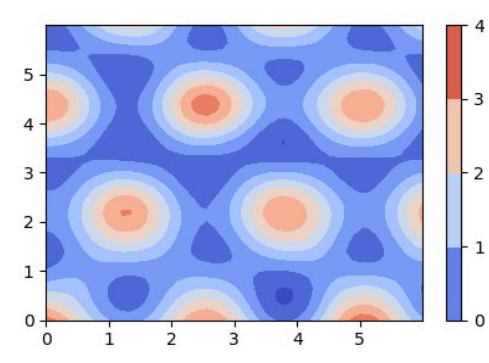

(b) Fe1SG

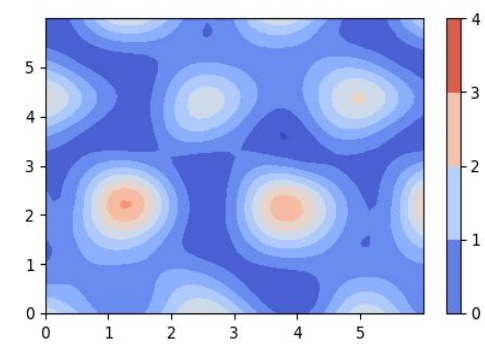

(c) Fe1SiG

Figure S4. Potential energy surface (PES) by relative sliding of two surfaces in three systems (a) Fe1PG, (b) Fe1SG, (c) Fe1SiG 


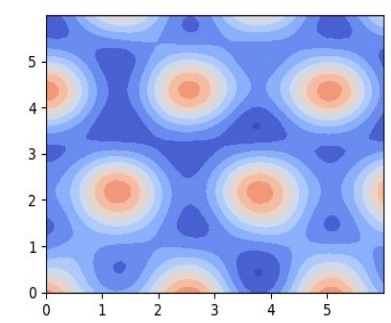

(a) Fe1PG

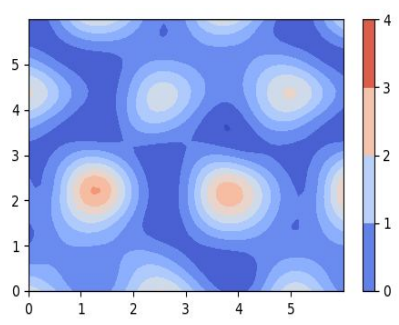

(e) Fe1SiG

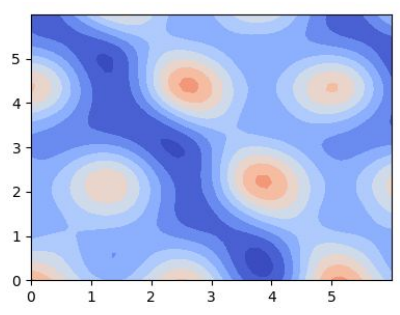

(b) $\mathrm{Fe} 2 \mathrm{PG}$

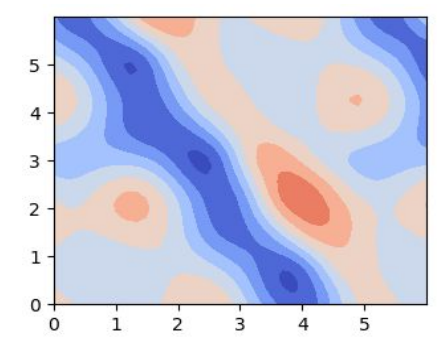

(f) $\mathrm{Fe} 2 \mathrm{SiG}$

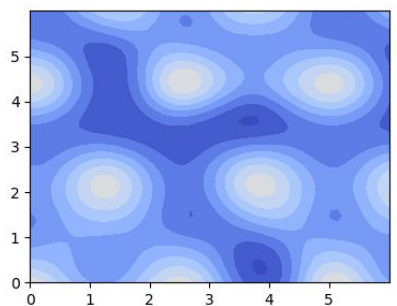

(c) Fe3PG

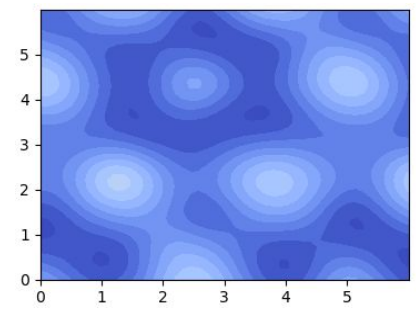

(d) Fe4PG

Figure S5. Potential energy surface (PES) by relative sliding of two surfaces in FePG $(\mathrm{a}-\mathrm{d})$, and $\mathrm{FeSiG}(\mathrm{e}-\mathrm{f})$.

Table S2. Bader charges of carbon and dopant atoms in FeDG systems for PES calculations. The $\mathrm{C} 1$ carbon is highlighted red in the table.

\begin{tabular}{rrrrrrrrrr}
\hline atom & \multicolumn{1}{c}{$\mathrm{FeG}$} & atom & $\mathrm{Fe} 1 \mathrm{BG}$ & $\mathrm{Fe} 1 \mathrm{PG}$ & $\mathrm{Fe} 1 \mathrm{SG}$ & atom & Fe4BG & Fe4PG & Fe4SG \\
\hline $\mathrm{C}$ & -0.14 & $\mathrm{C}$ & -0.08 & -0.08 & 0.05 & $\mathrm{C}$ & -0.21 & 0.16 & 0.18 \\
$\mathrm{C}$ & 0.15 & $\mathrm{C} 1$ & -0.58 & -0.65 & -0.18 & $\mathrm{C}$ & 0.07 & -0.08 & -0.06 \\
$\mathrm{C}$ & -0.15 & $\mathrm{C}$ & 0.20 & 0.04 & -0.06 & $\mathrm{C} 1$ & -0.63 & -0.64 & -0.11 \\
$\mathrm{C}$ & -0.14 & $\mathrm{C}$ & -0.01 & 0.17 & 0.02 & $\mathrm{C} 1$ & -0.64 & -0.64 & -0.13 \\
$\mathrm{C}$ & 0.22 & $\mathrm{C} 1$ & -0.56 & -0.69 & -0.21 & $\mathrm{C}$ & 0.03 & -0.10 & -0.10 \\
$\mathrm{C}$ & -0.14 & $\mathrm{C}$ & -0.08 & -0.22 & -0.18 & $\mathrm{C} 1$ & -0.63 & -0.60 & -0.10 \\
$\mathrm{C}$ & 0.15 & $\mathrm{C}$ & -0.06 & 0.03 & -0.05 & $\mathrm{C}$ & 0.09 & -0.07 & -0.07 \\
$\mathrm{C}$ & -0.13 & $\mathrm{C}$ & 0.18 & -0.06 & -0.08 & $\mathrm{C}$ & -0.21 & 0.16 & 0.18 \\
$\mathrm{C}$ & 0.15 & $\mathrm{C}$ & -0.15 & -0.02 & 0.14 & $\mathrm{C}$ & 0.07 & -0.08 & -0.06 \\
$\mathrm{C}$ & -0.15 & $\mathrm{C} 1$ & -0.61 & -0.69 & -0.22 & $\mathrm{C} 1$ & -0.63 & -0.64 & -0.11 \\
$\mathrm{C}$ & -0.14 & $\mathrm{C}$ & -0.06 & -0.06 & -0.06 & $\mathrm{C} 1$ & -0.64 & -0.64 & -0.13 \\
$\mathrm{C}$ & 0.22 & $\mathrm{C}$ & -0.21 & -0.04 & -0.07 & $\mathrm{C}$ & 0.03 & -0.10 & -0.10 \\
$\mathrm{C}$ & -0.14 & $\mathrm{C}$ & 0.21 & -0.05 & -0.16 & $\mathrm{C} 1$ & -0.63 & -0.60 & -0.10 \\
$\mathrm{C}$ & 0.15 & $\mathrm{C}$ & -0.15 & 0.14 & 0.14 & $\mathrm{C}$ & 0.09 & -0.07 & -0.07 \\
$\mathrm{C}$ & -0.14 & $\mathrm{C}$ & 0.15 & 0.15 & 0.15 & $\mathrm{C}$ & -0.21 & 0.16 & 0.18 \\
$\mathrm{C}$ & 0.15 & $\mathrm{C}$ & 0.13 & 0.04 & 0.05 & $\mathrm{C}$ & 0.07 & -0.08 & -0.06 \\
$\mathrm{C}$ & -0.15 & $\mathrm{C}$ & -0.03 & 0.17 & 0.17 & $\mathrm{C} 1$ & -0.63 & -0.64 & -0.11 \\
$\mathrm{C}$ & -0.14 & $\mathrm{C}$ & -0.08 & -0.08 & -0.06 & $\mathrm{C} 1$ & -0.64 & -0.64 & -0.13 \\
\hline
\end{tabular}




\begin{tabular}{rrrrrrrrrr}
\hline $\mathrm{C}$ & 0.22 & $\mathrm{C}$ & -0.20 & -0.03 & -0.07 & $\mathrm{C}$ & 0.03 & -0.10 & -0.10 \\
$\mathrm{C}$ & -0.14 & $\mathrm{C}$ & 0.18 & -0.05 & -0.04 & $\mathrm{C} 1$ & -0.63 & -0.60 & -0.10 \\
$\mathrm{C}$ & 0.15 & $\mathrm{C}$ & 0.06 & -0.05 & -0.01 & $\mathrm{C}$ & 0.09 & -0.07 & -0.07 \\
$\mathrm{C}$ & -0.14 & $\mathrm{C}$ & 0.06 & -0.07 & 0.05 & $\mathrm{C}$ & -0.21 & 0.16 & 0.18 \\
$\mathrm{C}$ & 0.15 & $\mathrm{C}$ & -0.04 & 0.16 & 0.17 & $\mathrm{C}$ & 0.07 & -0.08 & -0.06 \\
$\mathrm{C}$ & -0.15 & $\mathrm{C}$ & -0.15 & -0.08 & -0.05 & $\mathrm{C} 1$ & -0.63 & -0.64 & -0.11 \\
$\mathrm{C}$ & -0.14 & $\mathrm{C}$ & 0.08 & -0.09 & -0.06 & $\mathrm{C} 1$ & -0.64 & -0.64 & -0.13 \\
$\mathrm{C}$ & 0.22 & $\mathrm{C}$ & -0.21 & 0.14 & 0.20 & $\mathrm{C}$ & 0.03 & -0.10 & -0.10 \\
$\mathrm{C}$ & -0.14 & $\mathrm{C}$ & 0.15 & 0.05 & 0.13 & $\mathrm{C} 1$ & -0.63 & -0.60 & -0.10 \\
$\mathrm{C}$ & 0.15 & $\mathrm{C}$ & 0.12 & 0.17 & 0.10 & $\mathrm{C}$ & 0.09 & -0.07 & -0.07 \\
$\mathrm{C}$ & 0.03 & $\mathrm{C}$ & -0.09 & 0.07 & 0.06 & $\mathrm{~B} / \mathrm{P} / \mathrm{S}$ & 1.80 & 2.06 & 0.46 \\
$\mathrm{C}$ & 0.03 & $\mathrm{C}$ & -0.09 & -0.16 & 0.00 & $\mathrm{~B} / \mathrm{P} / \mathrm{S}$ & 1.80 & 2.06 & 0.46 \\
$\mathrm{C}$ & 0.03 & $\mathrm{C}$ & -0.03 & -0.07 & -0.14 & $\mathrm{~B} / \mathrm{P} / \mathrm{S}$ & 1.80 & 2.13 & 0.50 \\
$\mathrm{C}$ & 0.03 & $\mathrm{~B} / \mathrm{P} / \mathrm{S}$ & 1.78 & 1.99 & 0.42 & $\mathrm{~B} / \mathrm{P} / \mathrm{S}$ & 1.80 & 2.13 & 0.50 \\
\hline
\end{tabular}

\section{FPMD simulations for DG and FeDG}

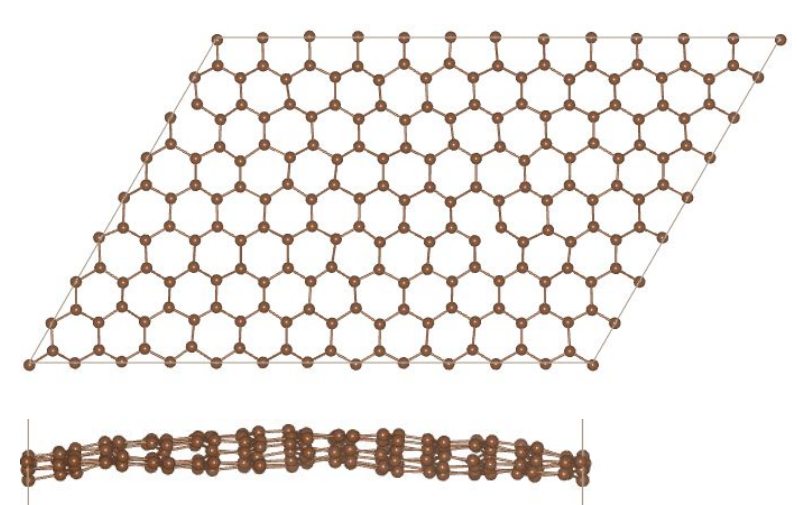

(a) Graphene

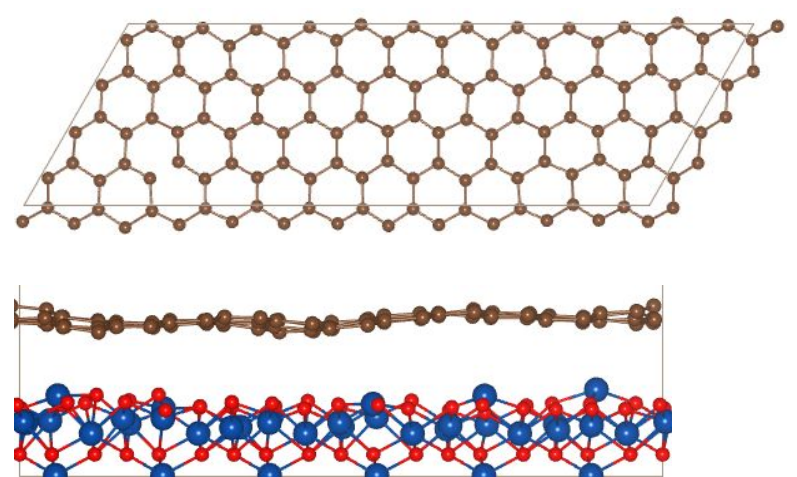

(b) $\mathrm{FeG}$

Figure S6. Top view and side view of graphene configuration after FPMD simulation at 1200 $\mathrm{K}$ and 10 ps. 


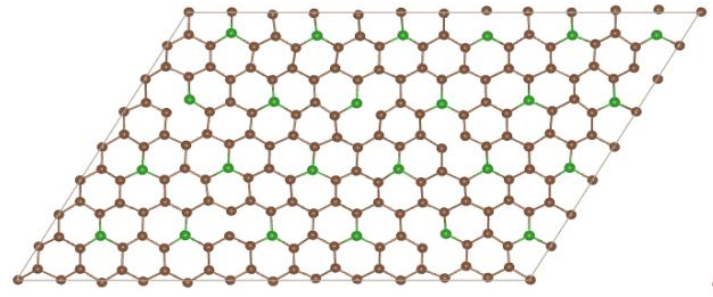

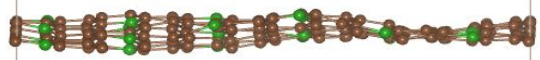

(a) 4BG

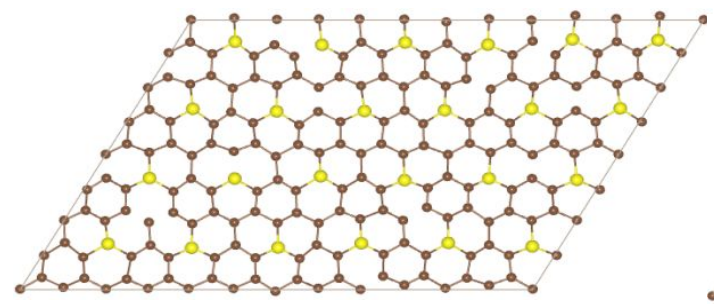

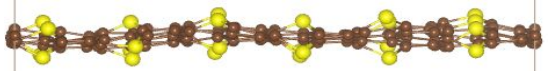

(c) $4 \mathrm{SG}$

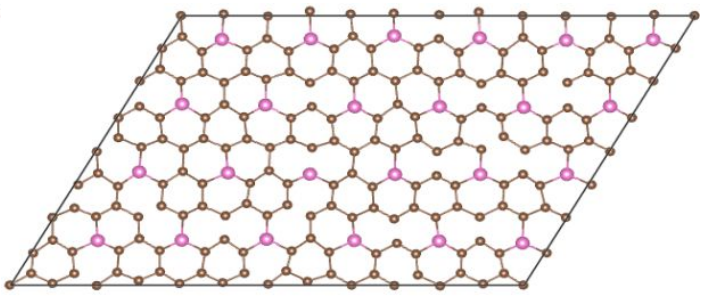

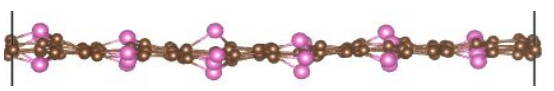

(b) 4PG

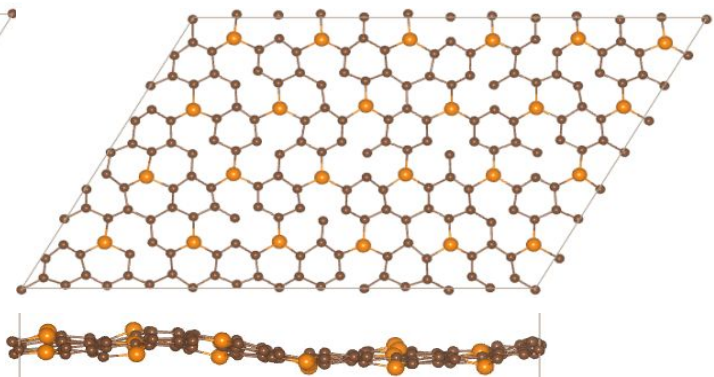

(d) $4 \mathrm{SiG}$

Figure S7. Output configurations of 4DG after FPMD simulation at $1200 \mathrm{~K}$ and $10 \mathrm{ps.}$
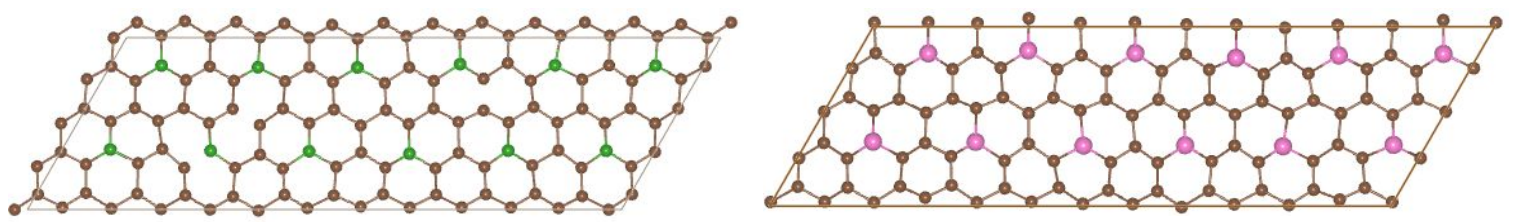

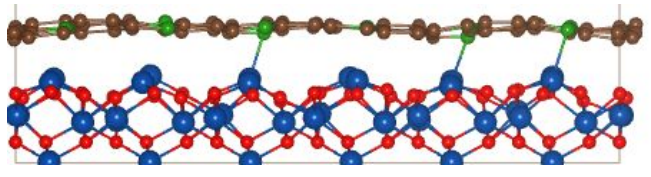

(a) $\mathrm{Fe} 4 \mathrm{BG}$
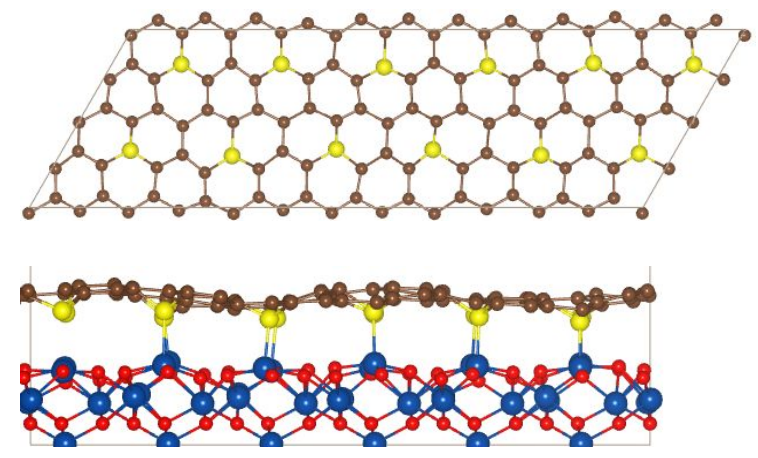

(c) Fe4SG

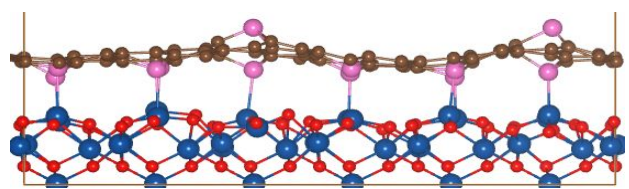

(b) Fe4PG
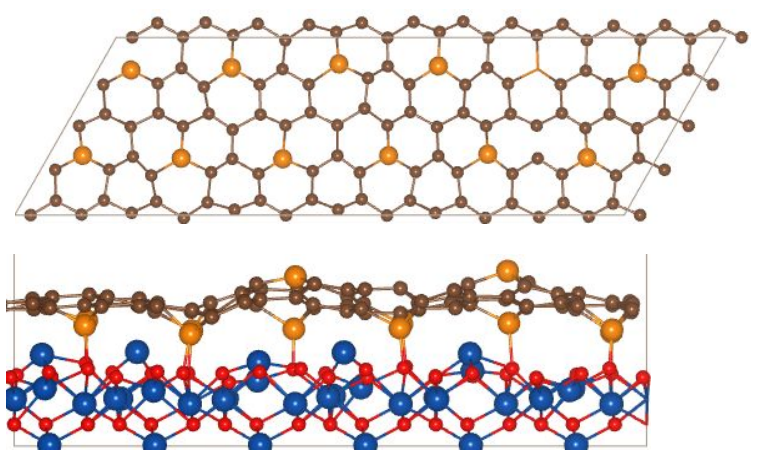

(d) $\mathrm{Fe} 4 \mathrm{SiG}$

Figure S8. Output configurations of Fe4DG after FPMD simulation at $1200 \mathrm{~K}$ and $10 \mathrm{ps.}$ 

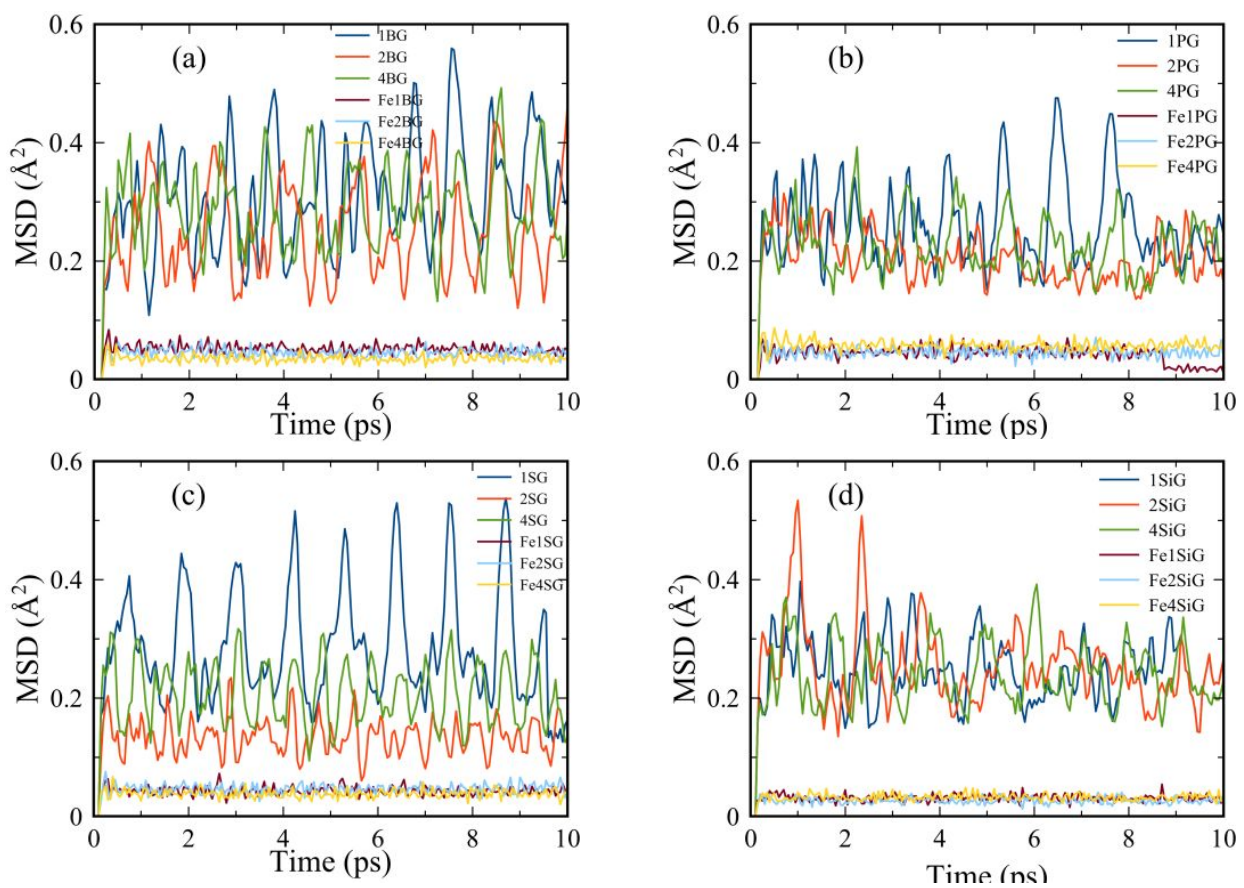

Figure S9. Mean square displacement (MSD) of carbon atoms in DG during the FPMD relaxation at $1200 \mathrm{~K}$. 